\title{
Automatic detection of pituitary microadenoma from magnetic resonance imaging using deep learning algorithms
}

Qingling Li ( $\square$ liqingl3@mail.sysu.edu.cn )

The Third Affiliated Hospital, Sun Yat-Sen University

Yanhua Zhu

The Third Affiliated Hospital, Sun Yat-Sen University

Minglin Chen

School of Electronics and Communication Engineering, Sun Yat-Sen University

Ruomi Guo

the Third Affiliated Hospital, Sun Yat-Sen University https://orcid.org/0000-0002-8207-8746

Hao Liu

School of Electronics and Communication Engineering, Sun Yat-Sen University

Tiecheng Zhang

The Second Affiliated Hospital, Harbin Medical University

\section{Huiquan Wen}

Third Affiliated Hospital of Sun Yat-Sen University

\section{Rong Gao}

The Third Affiliated Hospital, Sun Yat-Sen University

\section{Yuanpeng Nie}

The Third Affiliated Hospital, Sun Yat-Sen University

\section{Haicheng Li}

The Third Affiliated Hospital, Sun Yat-Sen University

\section{Jian Chen}

Third Affiliated Hospital of Sun Yat-sen University

\section{Guojun Shi}

The Third Affiliated Hospital, Sun Yat-Sen University

\section{Jun Shen}

Department of Urology, Department of Radiology, Department of Emergency Medicine, Department of respiratory medicine, Sun Yat-Sen Memorial Hospital, Sun Yat-Sen University https://orcid.org/00000001-7746-5285

\section{Wai Cheung}

University of California, San Diego

\section{Yulan Guo}


School of Electronics and Communication Engineering, Sun Yat-Sen University

\section{Yanming Chen}

the Third Affiliated Hospital of Sun Yat-Sen University

\section{Article}

Keywords: pituitary microadenoma, risk, CAD system, MR datasets

Posted Date: October 21st, 2020

DOI: https://doi.org/10.21203/rs.3.rs-94564/v1

License: (c) (1) This work is licensed under a Creative Commons Attribution 4.0 International License. Read Full License 


\title{
Automatic detection of pituitary microadenoma from magnetic resonance imaging using deep learning algorithms
}

\author{
Qingling $\mathrm{Li}^{1,8 \ddagger}$, Yanhua $\mathrm{Zhu}^{1 \ddagger}$, Minglin Chen ${ }^{2 \ddagger}$, Ruomi Guo ${ }^{3 \ddagger}$, \\ Hao Liu ${ }^{2}$, Tiecheng Zhang ${ }^{4}$, Huiquan $\mathrm{Wen}^{3}$, Rong $\mathrm{Gao}^{1}$, Yuanpeng $\mathrm{Nie}^{1}$, Haicheng $\mathrm{Li}^{1}$, \\ Jianning Chen ${ }^{5}$, Guojun $\mathrm{Shi}^{1}$, Jun Shen ${ }^{6}$, Wai Wilson Cheung ${ }^{7}$, \\ Yulan $\mathrm{Guo}^{2 *}$, Yanming Chen ${ }^{1 *}$
}

\footnotetext{
${ }^{1}$ Department of Endocrinology, The Third Affiliated Hospital, Sun Yat-Sen University, Guangzhou, China

${ }^{2}$ School of Eletronics and Communication Engineering, Sun Yat-Sen University, Guangzhou, China

${ }^{3}$ Department of Radiology, The Third Affiliated Hospital, Sun Yat-Sen University, Guangzhou, China

${ }^{4}$ Department of Magnetic Resonance, The Second Affiliated Hospital, Harbin Medical University, Harbin, China

${ }^{5}$ Department of Pathology, The Third Affiliated Hospital, Sun Yat-Sen University, Guangzhou, China

${ }^{6}$ Department of Radiology, The Sun Yat-Sen Memorial Hospital, Sun Yat-Sen University, Guangzhou, China

${ }^{7}$ Department of Pediatrics, University of California, San Diego, USA

${ }^{8}$ Department of VIP Medical Center, The Third Affiliated Hospital, Sun Yat-Sen University, Guangzhou, China
}

*These authors contributed equally to this work.

* Corresponding author:

Yanming Chen

Department of Endocrinology

The Third Affiliated Hospital, Sun Yat-Sen University

600 Tianhe Road, Guangzhou, China, 510630

Tel: (+86)020-85253108. E-mail: chyanm@mail.sysu.edu.cn

Yulan Guo

School of Eletronics and Communication Engineering, Sun Yat-Sen University,

135 Newport West Road, Guangzhou, China, 510275

Tel: (+86)020-84724135. E-mail: guoyulan@sysu.edu.cn

There is no conflicts of interest from the authors.

\section{Author contributions:}

Guarantors of integrity of entire study, Y.M.C., Y.L.G., study concepts/study design or data acquisition or data analysis/interpretation, all authors, manuscript drafting or manuscript revision for important intellectual content, all authors, approval of final version of submitted manuscript, all authors.

\section{Acknowledgments}

This study was funded by National Key R\&D Program of China (2017YFA0105803),

the General Program of National Natural Science Foundation of China (81770826, 61972435), the Key Area R\&D Program of Guangdong Province (2019B020227003),

the Natural Science Foundation of Guangdong Province (2019A1515011271), the Science and Technology Plan Projects of Guangzhou (202007040003),

and the Science and Technology Innovation Committee of Shenzhen Municipality (JCYJ20190807152209394).

We would like to thank all the patients and investigators for their participation in this study.

We also thank Chunkui Shao and Jing Wang (Professor, Department of Pathology and radiology,

The Third Affiliated Hospital of Sun Yat-sen University) and The Magnetic Resonance Department of Second Affiliated Hospital of Harbin Medical University for the help. 


\begin{abstract}
The risks of misdiagnosed pituitary microadenoma is high. We designed a convolutional neural network (CNN) based computer-aided diagnosis (CAD) system to retrospectively diagnose patients with pituitary microadenoma. A total 5,540 pituitary magnetic resonance (MR) images from 1,108 participants were recruited. MRI images were randomly stratified into non-overlapping cohorts (training set, validation set and test set) to establish five different CNN models. The best CNN model is the ResNet with a diagnostic accuracy of $94 \%$, which outperforms the diagnosis accuracy of our radiologists (64\%-85\%). The accuracy of our CAD system is further confirmed in additional MR datasets. The diagnostic accuracy of our ResNet model is comparable to the proficiency of a radiologist with 5-10 years' experience. In summary, this is the first report showing that the CAD system is a viable tool for diagnosing pituitary microadenoma. CAD system is applicable to radiology departments, especially in primary health care institutions. (148 words)
\end{abstract}




\section{INTRODUCTION}

Pituitary adenoma is one of the most common tumors in intracranial neoplasms and accounts for approximately $20 \%$ of all intracranial masses. Pituitary adenoma can result in various clinical symptoms, including infertility, diabetes and osteoporosis ${ }^{[1]}$. Previous radiology and autopsy studies have revealed a high prevalence of pituitary adenomas (10.4-31\%), and the vast majority (>99\%) are small microadenomas ${ }^{[2-4]}$. Most pituitary microadenomas are benign and non-functional tumors, which are diagnosed sporadically and easy to be missed. The pituitary is relatively small, and the anatomical structure of the pituitary gland differs among individuals. Manual analysis of magnetic resonance imaging (MRI) data is usually difficult, biased and time-consuming. It is reported that the diagnostic sensitivity for primary and recurrent pituitary microadenoma is only $47 \%$ and $39 \%$, respectively ${ }^{[5]}$. Therefore, accuracy of MRI-based diagnosis for pituitary microadenoma is still required to be improved. In our hospital, preliminary analysis indicates that the overall misdiagnosis rate of pituitary microadenoma among junior radiologist is about $47.6 \%$. The increase in the demand for senior radiologist is an inevitable trend, but the supply of radiologist has not increased proportionally. A shortage of radiologists, especially senior radiologists, restricts the continuity in radiology services and causes a delay in diagnosis, compromising the overall quality of service to patients ${ }^{[1,6]}$. Recently, we have encountered several missed diagnosis pituitary microadenomas in our hospital after further detailed diagnosis (Fig 1). Thus, timely and accurate detection of pituitary microadenoma is important to prevent potential sequelae in patients.

Artificial intelligence (AI) has the potential to revolutionize disease diagnosis and management by improving the diagnostic accuracy and reducing the workload of clinicians. The development of convolutional neural network $(\mathrm{CNN})$ has significantly improved the performance of image classification and object detection ${ }^{[7]}$. CNN has achieved great progresses in the diagnosis of breast cancer ${ }^{[8,9]}$, retinopathy ${ }^{[10,11]}$, and fibrotic lung disease ${ }^{[12]}$. Recently, several reports demonstrated that computer-aided diagnosis (CAD) system could accurately diagnose patients with pituitary tumors based on magnetic resonance (MR) images. However, the information for CAD-based diagnosis of pituitary microadenoma is scarce. Deep learning has dominated various computer vision areas since 2012 due to its effective representation ability ${ }^{[13]}$. In this work, a deep learning based CAD system is developed to provide an accurate and timely microadenoma diagnosis from enhanced pituitary MRI scans.

From a large number of networks, five CNNs (i.e., VGG ${ }^{[14]}$, GoogLeNet ${ }^{[15]}$, ResNeXt ${ }^{[16]}$, DenseNet ${ }^{[17]}$ and ResNet ${ }^{[18]}$ ) are chosen with the following considerations: First, these models are the most frequently used feature learning backbones for different computer vision applications. Second, they achieve state-of-the-art performance on several benchmarks such as ImageNet. Finally, their superiority has been demonstrated in many other medical image analysis tasks with promising performance. Results demonstrate that the CAD system developed in this study can reliably detect pituitary microadenoma in the sellar region. 


\section{RESULTS}

\section{Presentation of some misdiagnosed pituitary microadenoma in our hospital}

MRI is the gold standard for pituitary tumor detection. However, detection of microadenomas by MRI is restricted by its poor sensitivity. Our current research in applying AI deep learning algorithms to aide in diagnosing pituitary microadenoma were prompted by a few misdiagnosed cases in our hospital. A 43-years-old female patient was presented with diabetes, osteoporosis and recurrent cellulitis. Laboratory examination and functional test of this patient was consistent with clinical presentation of Cushing's disease (Tab S1). The patient underwent 3 rounds of enhanced MRI scanning over a period of 20 months but the radiologists could not detect the presence of pituitary adenoma (Fig 1a). The patient was treated with bromocriptine and cyproheptadine for two years, but the disease did not under effective control. Then, the inferior petrosal sinus sampling (IPSS) was performed on this patient and a functional microadenoma was detetced in the right pituitary gland of this patient. We performed an additional MRI examination on this patients and our senior radiologists were able to detect 2 pituitary microadenomas ( $5 \mathrm{~mm}$ and 3 $\mathrm{mm}$ in diameter, respectively) (Fig 1a). One week later, this patient underwent transsphenoidal pituitary microadenoma resection. Subsequent serum concentration of ACTH and free cortisol of this patient was normalized (Tab S1). Additional examples of misdiagnosed pituitary microadenomas in our hospital were presented (Fig 1b).

\section{Study design - baseline characters and base model selection}

AI learning requires a large number of samples to achieve superior diagnosis performance. The overall experiment design includes training, validation and testing. An explicit workflow is shown (Figure 2). A total of 5,540 images (2,190 with pituitary microadenoma and 3,350 with normal pituitary) were obtained from 1,108 patients who received pituitary MRI examination. Our CNN models were initially trained with 3,900 images $(1,370$ with pituitary microadenoma and 2,530 with normal pituitary) and validated with 975 images (330 with pituitary microadenoma and 645 with normal pituitary), subsequently the best CNN model was used to test with 665 images. The test dataset includes three parts. Test A was used to compare the diagnosis performance of a CNN model with human radiologists using 350 images (175 with pituitary microadenoma and 175 with normal pituitary). Test B was used to test the CNN model on false negative cases produced by radiologists (15 pituitary microadenoma images from 3 patients). Test $\mathrm{C}$ was used to test the universality and stability of the $\mathrm{CNN}$ model using images acquired from 3 hospitals (100 pituitary microadenoma images from each hospital). $5 \mathrm{CNN}$ models were trained and validated including VGG, GoogLeNet, ResNeXt, DenseNet and ResNet (Fig 3). After 500 epochs (iterations through the entire dataset), the training was stopped due to the absence of further improvement in both accuracy and cross-entropy loss (Fig 4). The final assessment on the test set was performed using the model obtained at epoch 500.

\section{Performance of five deep learning models}

These deep learning models achieved promising performance in diagnosing pituitary microadenoma. The performance of $5 \mathrm{CNN}$ models on pituitary MRI was shown (Table 1). The ResNet model achieved the best performance on the validation dataset, with an accuracy of $94.87 \%$, a sensitivity of $93.94 \%$, a specificity of $95.35 \%$, a PPV of $91.18 \%$, a NPV of $96.85 \%$, a F1-score of $92.54 \%$ and an area under the ROC curve (AUC) of $96.99 \%$ (Tab 1 and Fig S1). Hence, the ResNet model was employed in the following test set. 


\section{Diagnosis performance of the ResNet model versus radiologists}

An independent test set (with 350 images from 70 patients) were used to compare our ResNet model with radiologists in our hospital. Specifically, 6 radiologists were instructed to make a diagnosis for each image. The ResNet model diagnosis accuracy, sensitivity, specificity, PPV, NPV, and F1-score were 94.29\%, 97.14\%, 91.43\%, $91.89 \%, 96.97 \%$ and $94.44 \%$, respectively. In contrast, the best radiologist achieved a diagnosis accuracy of $84.57 \%$, a sensitivity of $85.14 \%$, a specificity of $84.00 \%$, a PPV of $84.18 \%$, a NPV of $84.97 \%$ and a F1-score of $84.66 \%$ (Tab $\mathrm{S} 2)$.

The receiver operating characteristics (ROC) curves (showing true positive rate and false positive rate for diagnosis performance on Test A were plotted in ROC space in Fig 5a) showed that the diagnosis performance of 6 radiologists lied below the ROC curves of the ResNet model and the AUC of ResNet was $97.14 \%$. Under the same false positive rate, the true positive rate of the ResNet model was higher than that of the radiologists (Fig 5a). A false positive result can cause unnecessary examination or excessive treatment. Moreover, a false negative result may result in irreversible loss. To account for these issues, weighted error scoring was incorporated during model evaluation and radiologist test. By assigning these penalty points to each decision made by the model and radiologists, their average error were computed. The ResNet model yielded an error of 7\% under this weighted error system, far below than the average weight error of $37.3 \%$ for 6 radiologists (Fig $5 b$ ). The differences in diagnostic performance (for both negative or positive likelihood ratios) between the ResNet model and human radiologists were statistically different (Fig 5c,d).

The exact breakdown of each radiologist's performance regarding the correlation of their predicted labels with the true labels was depicted as confusion matrices (Tab S3), the classification confusion matrices report the number of true positive, false positive, true negative, and false negative resulted for the ResNet model and radiologists. The ResNet model outperformed human radiologists based on this weighted error scale and on the ROC curve. Importantly, our ResNet model has an obvious technical advantages over radiologists in the detecting of pituitary microadenoma.

\section{Further confirmation the accuracy and reliability of ResNet model}

The double positive cases for microadenomas were diagnosed with radiologist and ResNet model. The timely diagnosis and treatment of positive cases could significantly improve disease prognosis. We sampled a number of double positive cases undergoing surgical treatment to further confirm the accuracy and reliability of our CAD system (Fig 6a and Tab S1). False negative result was far more serious than false positive cases as it may result in irreversible loss. Three cases that were misdiagnosed by our radiologists were selected for analysis. The false negative cases were relearn by our ResNet model. To our delight, the accuracy of ResNet model achieved 100\% and the diagnosis for each case was confirmed by subsequent pathological examination (Fig $6 \mathrm{~b}$ and Tab S1).

\section{Application of ResNet model for pituitary microadenoma detection}

We tested the effectiveness of ResNet model on detecting pituitary microadenoma in additional sets of samples. Pituitary MR images were obtained from 3 hospitals whereby each hospital provided 100 images from 20 patients with microadenoma. Six radiologists from each hospital were recruited to make the diagnosis of microadenomas based on those MRI. The accuracy of diagnosis of microadenoma was found to be positively correlated with the work experience of the individual radiologist as well as the allocated time for each image reading (Fig 7a, b). The 
diagnostic accuracy of pituitary microadenoma achieved by radiologists with professional experience over 10 years was above $80 \%$ and the diagnostic accuracy was greater than $80 \%$ when the allocated time for each image reading was longer than 12 seconds. In contrast, the pituitary microadenoma diagnosis accuracy of our ResNet model in 3 hospitals were $100 \%, 80 \%$ and $74.6 \%$, respectively. (Fig 7a). ResNet model was showed to have achieved compatible diagnostic performance of pituitary microadenoma with experienced radiologists.

\section{DISCUSSION}

The size of pituitary microadenomas are smaller than $10 \mathrm{~mm}$ diameters which accounts for $60 \%$ of all pituitary tumors and are often misdiagnosed because of the confusing array of symptoms they present ${ }^{[2,19]}$. Hormone-active adenomas exhibit complex pathophysiological disorders and accout for $46 \%$ to $85 \%$ of pituitary microadenomas ${ }^{[1}$, $2,19,20]$, while growth of non-functioning pituitary microadenomas occurs in about $10 \%$ of pituitary adenomas ${ }^{\text {[21]. }}$ Early diagnosis and appropriate treatment for functional or growth pituitary microadenoma are particularly important as it could minimize the damage in patients ${ }^{[22]}$. High-quality imaging of the sellar and parasellar region is critical to accurately diagnose of pituitary microadenoma ${ }^{[23]}$. However, the poor quality of MR images compound with the shortage of experienced radiologists make the manual analysis of MRI data difficult, biased and time-consuming. It has been reported that the current diagnostic sensitivity for primary and recurrent pituitary microadenoma is only $47 \%$ and $39 \%$, respectively ${ }^{[5,6]}$. Therefore, an accurate and efficient auxiliary diagnostic method for the diagnosis of pituitary microadenomas is highly needed.

Deep neural networks have been used to diagnose various diseases ${ }^{[9-12]}$. Specifically, most CNN model based research for pituitary diseases have been focused on pituitary macroadenoma ${ }^{[24-26]}$. Our work is the first study in applying CNN model for pituitary microadenomas diagnosis. The finding of a pituitary microadenoma by using MRI technique is rather low in the absence of amenorrhea and/or galactorrhea and/or infertility ${ }^{[27]}$. Compared to radiologists, CAD system can achieve consistent performance, efficient diagnosis and untired working. Therefore, it can assist radiologists by improving the diagnosis accuracy and reducing the workload. Inspired by previous studies in AI based tumor diagnosis. In this study, CNN models are proposed for diagnosis pituitary microadenoma. Our $\mathrm{CNN}$ models are comprised of two modules: pituitary detection and microadenoma diagnosis. The pituitary detection module has been developed for small object detection, which automatically localizes the pituitary region from 2D MRI slices. To augment for the fine-grained feature of pituitary, five patches only containing pituitary are cropped from MRI slices based on the output of the pituitary detection module. The microadenoma diagnosis module employes a CNN to detect pituitary microadenoma from five image patches. These results suggest that the proposed CNN models achieve high performance in accuracy, sensitivity, specificity for the diagnosis of pituitary microadenoma.

Five CNN models are trained and the best diagnosis performance is ResNet. The ResNet model yields satisfactory predictions on the validation dataset, with an accuracy of $94.87 \%$, a sensitivity of $93.94 \%$, and a specificity of $95.35 \%$. The ResNet model clearly outperforms radiologists based on this weighted scale and ROC curve. The weighted error rate for ResNet model is $7 \%$ versus $37.3 \%$ of the radiologists. Furthermore, to demonstrate the accuracy and reliability of the ResNet model, a number of double positive cases undergoing surgical treatment are selected. The efficacy of ResNet model has been evaluated in detecting the false negative cases, as false negative 
results are far more serious than false positive cases, which may result in irreversible loss. Comparing the negative and positive likelihood ratios achieve by ResNet model and human radiologists, the confidence interval shows that the ResNet model achieves better diagnosis performance, with a higher positive diagnosis rate and a lower false negative rate. Our ResNet model is then applied to learn the false negative cases by radiologists, the accuracy rate of the ResNet model to detect those false negative cases by radiologists is $100 \%$ as shown.

The CNN models are trained and validated with MR images from 3 different MRI machines. It is important that the CAD system achieves a robust generalization performance in diagnosis of pituitary microadenoma by using MR images generated by those different MRI machines. Additional MR images from 3 hospitals are used to further test the diagnostic accuracy of the ResNet model. The diagnostic accuracy for pituitary microadenomas by using ResNet model are $100 \%, 80 \%$ and $74.6 \%$, respectively in those 3 hospitals. Previous results suggest that CNN model performs better in the internal validation dataset than in the external test dataset ${ }^{[28,29]}$. Similarly, the ResNet model is observed to performed better on internal test dataset. Nevertheless, the ResNet model diagnostic accuracy for the external datasets is over $70 \%$ which is comparable to the diagnostic accuracy of a radiologist with professional experience of 5-10 years. In our study, the internal test dataset and the training dataset have similar MR images sources, whereas the images in the external test dataset come from different hospitals. Therefore, it is necessary to optimize CNN model with data collected from multiple centers. We showed that the system can be universally useful as the techniques described here can potentially be employed in a wide range of medical image analysis tasks across different hospitals ${ }^{[30]}$.

High-quality MR images are critical for the accurate diagnosis of pituitary adenoma. The increased use and accessibility of imaging techniques and improved spatial resolution of modern devices has led to a rise in incidental detection of pituitary microadenomas ${ }^{[3,6,18]}$. Importantly, accurate diagnosis of pituitary microadenoma is largely relied on the radiologist's professional experience. The diagnostic accuracy is steadily increased with the professional experience of individual radiologist as observed. Moreoever, with the prolonged reading time for each MR image, the diagnostic accuracy of radiologist can also be improved as shown. In this aspect, CAD system offers technical advantages over radiologists in the diagnosis of microadenoma. The performance of ResNet model is stable, and the accumulation of input data can theoretically improve the diagnostic ability of the CAD system ${ }^{[10-12]}$. The CNN-based CAD system can effectively reduce misdiagnosed rate for pituitary microadenoma, especially in primary healthcare providers whereby there is a lack of experienced radiologists.

This is the first report in applying $\mathrm{CNN}$ for the diagnosis of pituitary microadenoma. Our results shows that the ResNet model can provide a diagnostic accuracy comparable to experienced radiologists with marginal deployment costs. We acknowledge that there are several limitations for the current investigation. Firstly, this is a retrospective study. Further validation with larger and prospective studies are needed prior to actual clinical application. Secondly, although a total of 5,540 MR images have been used to design, train, and test in AI system, the input dataset was still insufficient. Previous studies have shown that robustness and accuracy of deep learning machine increases proportionally with the amount of input dataset ${ }^{[18,31]}$.

In conclusion, results of our research highlights the potential applications of using AI to benefit the clinical diagnosis of patients with pituitary microadenoma. As computational processing power continues to increase, and deep learning algorithms continue to surpass gold diagnosis standard, machine learning for the diagnosis of pituitary 
microadenoma will serve as an important component in improving patient care and outcomes.

\section{MATERIALS AND METHODS}

\section{Ethical Approval}

This study is approved by the research ethics committee of the Institute of Basic Research in Clincial Medicine, The Third Affiliated Hospital of Sun Yat-Sen University ([2020]02-089-01). This research is registered at the Chinese Clinical Trials Registry (http://www.chictr.org.cn/index. aspx) with the number ChiCTR2000032762.

\section{Data collection and pre-procession of MRI data}

A total of 1,108 patients with MR images (GE, Philips company) were selected from retrospective cohorts of patients from 3 hospitals from January 2012 to December 2019. All MR images for AI learning was randomly assigned regardless of the type of MRI machine or the manufacturer. The internal dataset which was collected from The Third Affiliated Hospital of Sun Yat-Sen University (hospital 1) was mainly used for training and validation. The external dataset was collected from The Sun Yat-Sen Memorial Hospital of Sun Yat-Sen University (hospital 2) and The Second Affiliated Hospital of Harbin Medical University (hospital 3). All MR imaging was performed as part of patients' routine clinical care. There were no exclusion criteria based on age, gender, or ethnicity. Local electronic medical record databases for diagnoses of pituitary microadenoma (438 patients, 2,190 images) and normal (670 controls, 3,350 images) were searched to initially assigned images. Enhanced pituitary scan was downloaded with a standard image format according to manufacurer's software and instructions. For the analysis of all MR images, 3 radiologists were initially screened for quality control by removing all low quality or unreadable scans. The images were diagnosed by 3 experienced radiologists who were consulted in case of disagreement before $\mathrm{CNN}$ models training, and 5 clearest images of the pituitary coronal position from each patient were selected. The process of data pre-procession was shown in Fig 2. Finally, these data were randomly divided into training, validation or test set for further analysis. In order to account for any grading errors, the evaluation set was also checked by two senior endocrinologists.

\section{Classification of the test dataset}

The test dataset was divided into 3 parts (test $\mathrm{A}$, test $\mathrm{B}$ and test $\mathrm{C}$ ). Test $\mathrm{A}$ was used to compare the diagnostic accurary of ResNet model versus radiologists. Test B was used to test the diagnosis accuracy in misdiagonosed cases by using ResNet model. Test $\mathrm{C}$ was used to verify the universality and stability of ResNet model. Six radiologists were recruited to read the same MRI images. The professional experience of these 6 radiologists were listed as following; 2 radiologists with more than 10 years professional experience, 2 radiologists with 5 - 10 years professional experience and 2 radiologists with less than 5 years professional experience.

\section{Overview of the CNN model}

The architecture of the CNN model was shown in Fig 3. Before entering our CNN model, 5 axial image slices containing pituitary gland coronal were selected from the whole MRI scan by radiologists. Our CNN model was comprised of two modules: pituitary detection and microadenoma diagnosis. In the pituitary detection module, the Faster R-CNN network ${ }^{[32]}$ was employed to locate pituitary region from each MRI slice. The network processed the input MRI slice to generated classification and regression maps which were further used to extract the pituitary bounding box in MRI slice. Five pituitary bounding boxes were obtained by the output of the network. Based on the 
five pituitary bounding boxes, five patches containing pituitary were cropped from the corresponding MRI slices. Before entering the microadenoma diagnosis module, 5 pituitary patches were resized to $256 \times 256$, and then stacked to form an image with five channels. In the microadenoma diagnosis module, various CNNs (i.e. VGG ${ }^{[14]}$, GoogLeNet ${ }^{[15]}$, ResNeXt ${ }^{[16]}$, DenseNet ${ }^{[17]}$ and ResNet ${ }^{[18]}$ ) were used in microadenoma diagnosis. The CNN model extracted the hierarchical features of the input image, and produced the presence probability $p$ of microadenoma through softmax layer. The input MRI was considered abnormal when $p>$ or $=0.5$ and normal otherwise.

\section{Biochemical tests and histopathological}

At screening, patients underwent a comprehensive physical examination, pituitary MRI and fasting blood sampling for biological measurements, including HbAlc which was measured by D-10 hemoglobin Testing Program (Bio-Rad) with high performance liquid chromatography (HPLC). Other relevant serum chemistry such as thyroid stimulating hormone (TSH), free thyroxine (FT4), free triiodothyronine (FT3), luteinizing hormone (LH), follicle stimulating hormone $(\mathrm{FSH})$, total testosterone (TT), estradiol $\left(\mathrm{E}_{2}\right)$, prolactin (PRL) and progesterone (PRGE) were measured by chemiluminescence method (Abbott, USA, I2000). Plasma cortisol, growth hormone (GH), insulin-like growth factor-1(IGF-1) and 24-hour urinary free cortisol (PZC24) were measured by chemiluminescence method (Siemens, Germany, Ceutaur XP). Serum adrenocorticotropic hormone (ACTH) was measured by chemiluminescence method (Siemens, Germany, IMI2000), patients underwent appropriate screening for hypercortisolism (basal cortisol level of 8 am, 4 pm and midnight serum cortisol, 1-mg and/or 8-mg dexamethasone suppression test and the suppression of cortisol greater than $50 \%$ basal cortisol level was considered a positive result) ${ }^{[33-35]}$. For this study, 6 positive and false negative patients undergoing transsphenoidal resection of the pituitary microadenoma were included (Fig 6). After the surgical operation, the tumor tissues from patients were used for hematoxylin and eosin (HE) staining as well as immunohistochemistry (ACTH, GH, FSH, PRL, TSH, LH) staining to determine the type of pituitary microadenoma ${ }^{[29,34]}$.

\section{Model testing and statistical analysis}

The 3,900 images of training set were collected (from 780 patients) and used to train the CNN model, while another 975 images (from 195 patients) of the validation set were used in the best CNN model selection for the following analysis. After picking up the best CNN model (ResNet model) on the validation set, the test set was used to evaluate the generalization ability of the ResNet model on the unseen data. During test, the images were diagnosed for the presence of microadenomas by ResNet model, if the probability was larger than the threshold value (i.e. p $>$ or $=0.5$ ). To measure the diagnostic performance of the ResNet model and the radiologists, the following metrics were used: accuracy, sensitivity, specificity, positive predictive value (PPV), negative predictive value (NPV), F1score, weighted error, positive likelihood ratio (PLR) and negative likelihood ratio (NLR) ${ }^{[10,24]}$. These metrics formulated as follows: accuracy $=(\mathrm{TP}+\mathrm{TN}) /(\mathrm{TP}+\mathrm{FP}+\mathrm{TN}+\mathrm{FN})$, sensitivity $=\mathrm{TP} /(\mathrm{TP}+\mathrm{FN})$, specificity $=\mathrm{TN} /(\mathrm{TN}$ $+\mathrm{FP}), \mathrm{PPV}=\mathrm{TP} /(\mathrm{TP}+\mathrm{FP}), \mathrm{NPV}=\mathrm{TN} /(\mathrm{TN}+\mathrm{FN}), \mathrm{F} 1$-score $=(2 * \mathrm{TP}) /(2 * \mathrm{TP}+\mathrm{FP}+\mathrm{FN})$, weighted Error $=$ $(2 * \mathrm{FP}+1 * \mathrm{FN}) /(\mathrm{TP}+\mathrm{FP}+\mathrm{TN}+\mathrm{FN}), \mathrm{PLR}=$ Sensitivity / (1-Specificity), NLR = (1-Sensitivity) / Specificity, where TP, TN, FP and FN represented the number of true positives, true negatives, false positives and false negatives, respectively. In addition, ROC and AUC ${ }^{[10]}$ were used to test the robustness of the probability threshold selection of the CNN model. ROC curves were used to plot the true positive rate (sensitivity) versus the false positive rate (1specificity). The area under the ROC curve was a measure of performance for pituitary microadenoma diagnosis. 
Weighted error scoring was used to reflect the fact that a false negative result was more detrimental than a false positive result, the penalties included an error scored of 2 for false negative scored cases and an error scored of 1 for false positive scored cases ${ }^{[24]}$. The positive likelihood ratio was defined as the true positive rate over the false positive rate, so that an increasing likelihood ratio larger than 1 indicated increasing probability that diagnosis adenoma. The negative likelihood ratio was defined as the false negative rate over the true negative rate, so that a decreasing likelihood ratio less than 1 indicated increasing probability that absence of adenoma ${ }^{[10]}$. All these metrics were calculated using the Python software (https://www.python.org/), and their 95\% confidence intervals (CIs) were derived based on normal approximation interval ${ }^{[10]}$. Continuous variables were presented as mean \pm standard deviation (SD), for continuous values, one-way ANOVA was used to determine statistical significance. The statistical analysis was conducted by SPSS software (version 25.0). The $\mathrm{p}$ value $<$ or $=0.05$ was considered statistical significance.

\section{Supplemental Information}

Supplemental Information includes one figure and 3 tables.

\section{Reporting Summary}

Further information on research design is available in the Nature Research Reporting Summary linked to this article.

\section{Data Availability}

Excel files containing raw data included in the main figures and tables can be found in the Source Data File in the article. All other data are available in the Article and Supplementary Information. All other data including the imaging data can be provided upon reasonable request to the corresponding author.

\section{Code Availability}

The software and code of the proposed method have been separated into two files and are available as Supplementary Software files. https://github.com/MinglinChen94/PituitaryMicroadenomaDiagnosis 


\section{REFERENCES}

1. Ostrom, Q. T. et al. CBTRUS Statistical Report: Primary Brain and Other Central Nervous System Tumors Diagnosed in the United States in 2012-2016. Neuro Oncol. 21(Suppl 5), v1-v100 (2019).

2. Lake, M. G., Krook, L. S. \& Cruz, S. V. Pituitary Adenomas: an overview. Am Fam Physician. 88, 319-27 (2013).

3. Paschou, S. A., Vryonidou, A. \& Goulis, D. G. Pituitary Incidentalomas: A guide to assessment, treatment and follow-up. Maturitas. 92, 143-149 (2016).

4. Buurman, H. \& Saeger, W. Subclinical adenomas in postmortem pituitaries: classification and correlations to clinical data. Eur J Endocrinol. 154, 753-8 (2006).

5. Wang, H. et al. PET/MRI in the Diagnosis of Hormone-Producing Pituitary Microadenoma: A Prospective Pilot Study. J Nucl Med. 59, 523-528 (2018).

6. Yan, P. F. et al. Accuracy of conventional MRI for preoperative diagnosis of intracranial tumors: A retrospective cohort study of 762 cases. Int J Surg. 36,109-117 (2016).

7. Anwar, S. M. et al. Medical Image Analysis using Convolutional Neural Networks: A Review. J Med Syst. 42, 226 (2018).

8. Zheng, X. et al. Deep learning radiomics can predict axillary lymph node status in early-stage breast cancer. Nat Commun. 11, 1236 (2020).

9. Zhou, L. Q. et al. Lymph Node Metastasis Prediction from Primary Breast Cancer US Images Using Deep Learning. Radiology. 294, 19-28 (2020).

10. Kermany, D. S. et al. Identifying Medical Diagnoses and Treatable Diseases by Image-Based Deep Learning. Cell. 172, 1122-1131 (2018).

11. Varadarajan, A. V. et al. Predicting optical coherence tomography-derived diabetic macular edema grades from fundus photographs using deep learning. Nat Commun. 11, 130 (2020).

12. Walsh, S. L. F. et al. Deep learning for classifying fibrotic lung disease on high-resolution computed tomography: a case-cohort study. Lancet Respir Med. 6, 837-845 (2018).

13. Krizhevsky, A., Sutskever, I. \& Hinton, G. E. ImageNet Classification with Deep Convolutional Neural Networks. International Conference on Neural Information Processing Systems. 25 (2012).

14. Simonyan, K. \& Zisserman, A. Very deep convolutional networks for large-scale image recognition. The International Conference on Learning Representations (ICLR). 2015.

15. Szegedy, C. et al. Going deeper with convolutions. International Conference on Computer Vision and Pattern Recognition. 1-9 (2015).

16. Xie, S. et al. Aggregated Residual Transformations for Deep Neural Networks. International Conference on Computer Vision and Pattern Recognition. 1492-1500 (2017) .

17. Huang, G. et al. Densely Connected Convolutional Networks. International Conference on Computer Vision and Pattern Recognition. 4700-4708 (2017).

18. He, K. et al. Deep Residual Learning for Image Recognition. International Conference on Computer Vision and Pattern Recognition. 770-778 (2016).

19. Gruppetta, M., Mercieca, C. \& Vassallo, J. Prevalence and incidence of pituitary adenomas: a population based study in Malta. Pituitary. 16, 545-53 (2013). 
20. Ezzat, S. et al. The prevalence of pituitary adenomas: a systematic review. Cancer. 101, 613-9 (2004).

21. Molitch, M. E. Pituitary tumours: pituitary incidentalomas. Best Pract Res Clin Endocrinol Metab. 23, 667-75 (2009).

22. Drummond, J., Roncaroli, F., Grossman, A. B. \& Korbonits, M. Clinical and Pathological Aspects of Silent Pituitary Adenomas. J Clin Endocrinol Metab. 104, 2473-2489 (2019).

23. Bashari, W. A. et al. Modern imaging of pituitary adenomas. Best Pract Res Clin Endocrinol Metab. 33, 101278 (2019).

24. Qian, Y. et al. A novel diagnostic method for pituitary adenoma based on magnetic resonance imaging using a convolutional neural network. Pituitary. 23, 246-252 (2020).

25. Ugga, L. et al. Prediction of high proliferative index in pituitary macroadenomas using MRI-based radiomics and machine learning. Neuroradiology. 61, 1365-1373 (2019).

26. Fan, Y. et al. Preoperative Noninvasive Radiomics Approach Predicts Tumor Consistency in Patients With Acromegaly: Development and Multicenter Prospective Validation. Front Endocrinol (Lausanne). 28, 403 (2019).

27. Souter, I. et al. Prevalence of hyperprolactinemia and abnormal magnetic resonance imaging findings in a population with infertility. Fertil Steril. 94, 1159-62 (2010).

28. Long, E. et al. An artificial intelligence platform for the multihospital collaborative management of congenital cataracts. Nature Biomedical Engineering. 0024 (2017).

29. Wu, Q. et al. Expert-level Diagnosis of Nasal Polyps Using Deep Learning on Whole-slide Imaging. J Allergy Clin Immunol. 145, 698-701 (2020).

30. Shen, D., Wu, G. \& Suk, H. I. Deep Learning in Medical Image Analysis. Annu Rev Biomed Eng. 19, 221-248 (2017).

31. Lecun, Y., Bengio, Y. \& Hinton, G. Deep learning. Nature. 521, $436-44$ (2015) .

32. Ren, S. et al. Faster R-CNN: Towards Real-Time Object Detection with Region Proposal Networks. IEEE Trans Pattern Anal Mach Intell. 39, 1137-1149 (2017).

33. Yan, J. et al. Liraglutide, Sitagliptin, and Insulin Glargine Added to Metformin: The Effect on Body Weight and Intrahepatic Lipid in Patients With Type 2 Diabetes Mellitus and Nonalcoholic Fatty Liver Disease. Hepatology. 69, 2414-2426 (2019).

34. Gezer, E. et al. Cushing's disease due to a pituitary adenoma as a component of collision tumor: A case report and review of the literature. J Med Case Rep. 14, 59 (2020).

35. Molitch ME. Diagnosis and Treatment of Pituitary Adenomas: A Review. JAMA. 317, 516-524 (2017). 
Table 1. Performance of 5 CNN Models.

\begin{tabular}{|c|c|c|c|c|c|c|}
\hline & \multirow{2}{*}{ Accuracy } & \multirow{2}{*}{ F1 Score } & \multicolumn{2}{|c|}{ Positive Metrics } & \multicolumn{2}{|c|}{ Negative Metrics } \\
\hline & & & Sensitivity & PPV & Specificity & NPV \\
\hline VGG & 0.9026 & 0.8550 & 0.8485 & 0.8615 & 0.9302 & 0.9231 \\
\hline & $(0.8609 \sim 0.9442)$ & $(0.7947 \sim 0.9153)$ & $(0.7620 \sim 0.9350)$ & $(0.7776 \sim 0.9455)$ & $(0.8863 \sim 0.9742)$ & $(0.8773 \sim 0.9689)$ \\
\hline GoogLeNet & $\begin{array}{c}0.9128 \\
(0.8732 \sim 0.9524)\end{array}$ & $\begin{array}{c}0.8722 \\
(0.8154 \sim 0.9289)\end{array}$ & $\begin{array}{c}0.8788 \\
(0.8000 \sim 0.9575)\end{array}$ & $\begin{array}{c}0.8657 \\
(0.7840 \sim 0.9473)\end{array}$ & $\begin{array}{c}0.9302 \\
(0.8863 \sim 0.9742)\end{array}$ & $\begin{array}{c}0.9375 \\
(0.8956 \sim 0.9794)\end{array}$ \\
\hline ResNeXt & $\begin{array}{c}0.9385 \\
(0.9047 \sim 0.9722)\end{array}$ & $\begin{array}{c}0.9077 \\
(0.8579 \sim 0.9575)\end{array}$ & $\begin{array}{c}0.8939 \\
(0.8197 \sim 0.9682)\end{array}$ & $\begin{array}{c}0.9219 \\
(0.8561 \sim 0.9876)\end{array}$ & $\begin{array}{c}0.9612 \\
(0.9279 \sim 0.9945)\end{array}$ & $\begin{array}{c}0.9466 \\
(0.9081 \sim 0.9851)\end{array}$ \\
\hline DenseNet & $\begin{array}{c}0.9385 \\
(0.9047 \sim 0.9722)\end{array}$ & $\begin{array}{c}0.9104 \\
(0.8621 \sim 0.9588)\end{array}$ & $\begin{array}{c}0.9242 \\
(0.8604 \sim 0.9881)\end{array}$ & $\begin{array}{c}0.8971 \\
(0.8248 \sim 0.9693)\end{array}$ & $\begin{array}{c}0.9457 \\
(0.9066 \sim 0.9848)\end{array}$ & $\begin{array}{c}0.9606 \\
(0.9268 \sim 0.9945)\end{array}$ \\
\hline ResNet & $\begin{array}{c}0.9487 \\
(0.9178 \sim 0.9797)\end{array}$ & $\begin{array}{c}0.9254 \\
(0.8809 \sim 0.9699)\end{array}$ & $\begin{array}{c}0.9394 \\
(0.8818 \sim 0.9970)\end{array}$ & $\begin{array}{c}0.9118 \\
(0.8443 \sim 0.9792)\end{array}$ & $\begin{array}{c}0.9535 \\
(0.9171 \sim 0.9898)\end{array}$ & $\begin{array}{c}0.9685 \\
(0.9381 \sim 0.9989)\end{array}$ \\
\hline
\end{tabular}

Note.-Unless otherwise specified, data in parentheses are percentages. Numbers in parentheses are the range of $95 \%$ confidence intervals. CNN: convolutional neural network. F1 score: the harmonic mean of PPV and sensitivity. PPV: positive predictive value. NPV: negative predictive value. 
Fig 1. Representation of misdiagnosed pituitary microadenoma. (a) We showed 4 consecutive pituitary MRI scans over a period of 20 months in a misdiagnosed patient. The radiologist did not pick up the pituitary adenoma during the first 3 times MRI examination. Subsequent ACTH examination of the inferior petrosal sinus localized a functional microadenoma in the region of right pituitary gland. On the 4th MRI scanning, the radiologist detected two pituitary microadenomas. (b) Additional 3 cases of misdiagnosed pituitary microadenoma were shown. Pituitary microadenoma is characterized by variable morphology and prone to misdiagnosis. Patient 1 had a very small pituitary microadenoma with diameter $<3 \mathrm{~mm}$. Patient 2 had an irregularly shaped pituitary microadenoma. Patient 3 had two pituitary microadenomas $(2.8 \mathrm{~mm}$ and $6.1 \mathrm{~mm}$, respectively) and the smaller microadenoma was misdiagnosed. The comprehensive clinical data for patients were listed in supplementary table 1. ACTH: Adrenocorticotropic Hormone. MRI: magnetic resonance imaging. T1WI-COR: T1 weighted imaging-coronal. MRI bar $=5 \mathrm{~mm}$. The yellow arrow and the area inside the red circle are adenomas.

Fig 2. Workflow diagram showing for overall experimental design. All pituitary MRI was performed as part of patients' routine clinical care. There were no exclusion criteria based on age, gender, or race. MRI: magnetic resonance imaging.

Fig 3. Overview of proposed CAD system. (a) Five coronal images of the pituitary gland were firstly selected from the original MRI scan by radiologists. Then, the selected MR images were fed into our CAD system for automatic diagnosis. The proposed CAD system consisted two modules: (b) the pituitary detection module employed Faster-RCNN to localize the pituitary region in cerebral MRI. It processed the MRI image using multiple convolutional layers and produced two maps (where the classification map was used to predict the center and the regression map was used to refine the height and width of the rectangle box) for predicting the rectangle box which represented the pituitary region. The pituitary rectangle region was cropped and stacked before feeding into (c) which employed one of CNNs comprising multiple convolutional layers (i.e. VGG, GoogLeNet, ResNeXt, DenseNet, ResNet) to extract the feature. A softmax layer was employed to transform the feature into the presence probability of microadenoma. The images were diagnosed as microadenoma if the $\mathrm{p}$ value $>$ or $=0.5$. CAD: computer-aided diagnosis. MRI: magnetic resonance imaging. MR: magnetic resonance. CNN: convolutional neural network.

Fig 4. Performance of ResNet model for training and validation datasets. (a). Accuracy curves of ResNet model on training and validation datasets. (b). Cross entropy loss curves of ResNet model on training and validation datasets. After 500 epochs (iterations through the entire dataset), the training and validation datasets were stopped due to the absence of further improvement in both accuracy and cross-entropy.

Fig 5. ResNet model outperformed human radiologists in diagnosing pituitary microadenoma. (a) ROC and AUC: Receiver operating characteristics (ROC) curves plotted the true positive rate (sensitivity) versus the false positive rate (1- specificity). The ROC showed that the ResNet model outperformed 6 radiologists. The AUC (area under ROC curve) of ResNet was $97.14 \%$. (b) Weighted error results based on penalties, the penalties included an error scored of 2 for false negative scored cases and an error scored of 1 for false positive scored cases, the ResNet model yielded a score of 7\% versus the radiologists was $37.3 \%$. (c \& d) The negative likelihood ratio and the positive likelihood ratio: The negative likelihood ratio was defined as the false negative rate over the true negative rate, so that a decreasing likelihood ratio < 1 indicated increasing probability the absence of microadenoma. The positive likelihood ratio was defined as the true positive rate over the false positive rate, so that an increasing likelihood ratio $>1$ indicated increasing probability the diagnosis of microadenoma. The confidence intervals showed that the ResNet model demonstrated statistically better screening performance in both negative likelihood ratio and positive likelihood ratio relative to human radiologists. Radiologist $1 \& 2$ : with $>10$ years professional experience, radiologist $3 \& 4$ : with 5 - 10 years professional experience, radiologist $5 \& 6$ : with $<5$ years.

Fig 6. The MRI and pathologic images of double positive and false negative cases. (a, b) 3 double positive and 3 false negative cases, which were functional pituitary microadenoma, as confirmed by subsequent pathological examination. (b) ResNet model learning 3 false negative cases from radiologists: showing the model diagnosis performance was $100 \%$ accuracy. The comprehensive clinical data for patients were listed in Supplementary table 1. MRI: magnetic resonance imaging. AI: Artificial intelligence. HE: hematoxylin and eosin. ACTH: adrenocorticotropic hormone. GH: growth hormone. TSH: thyroid stimulating hormone. PRL: prolactin. MR bar $=5 \mathrm{~mm}$. Pathology bar $=100 \mu \mathrm{m}$. The yellow arrow indicates a pituitary micro-adenoma.

Fig 7. Application of ResNet model in diagnosing pituitary microadenoma. MR images were obtained from 3 hospitals. (a) The diagnosis accuracy rate was significantly increased in senior radiologist and the ResNet model outperformed the radiologists with 5-10 years professional experience in 3 hospitals. (b) The accuracy for diagnosis rate was significantly increased by radiologist with longer reading times. Bar means $p$ value $<$ or $=0.05$ for comparison of two groups. AI: Artificial intelligence. MR: magnetic resonance. 
Fig 1

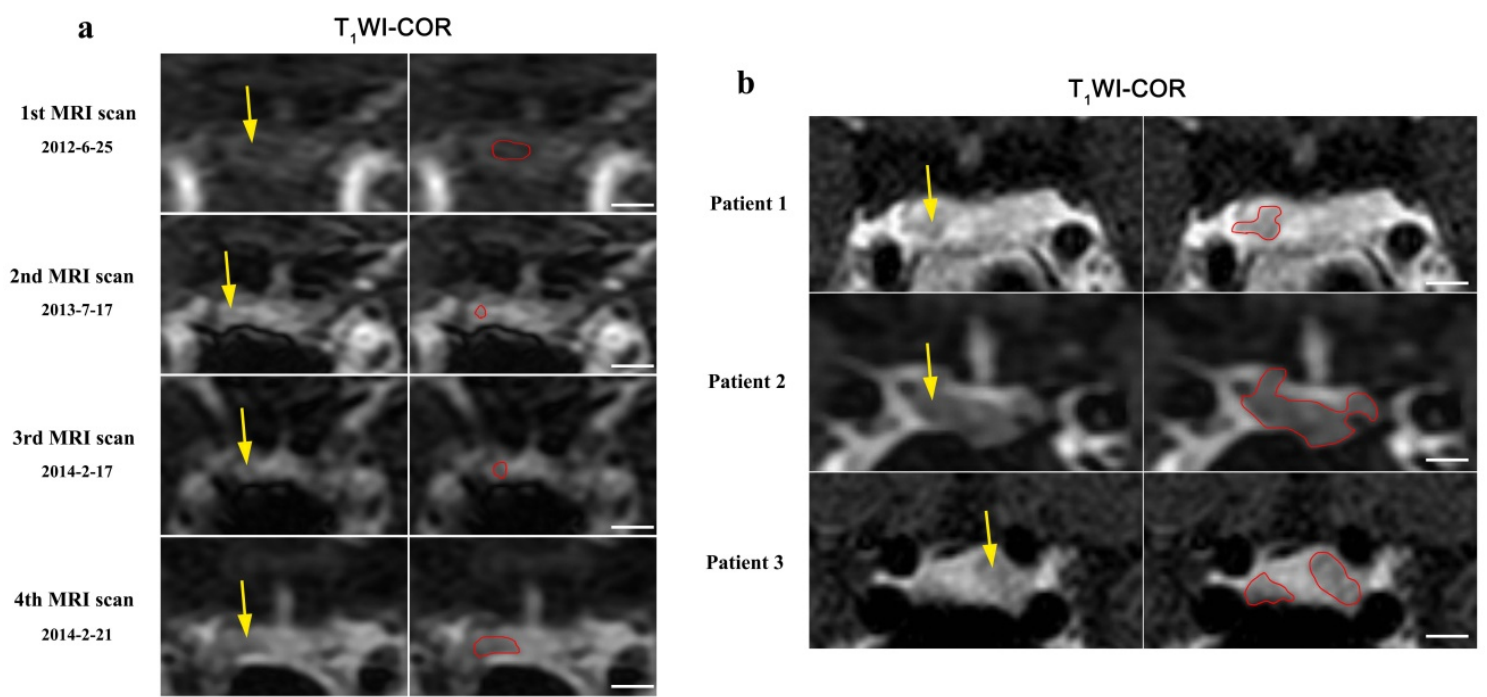


Fig 2

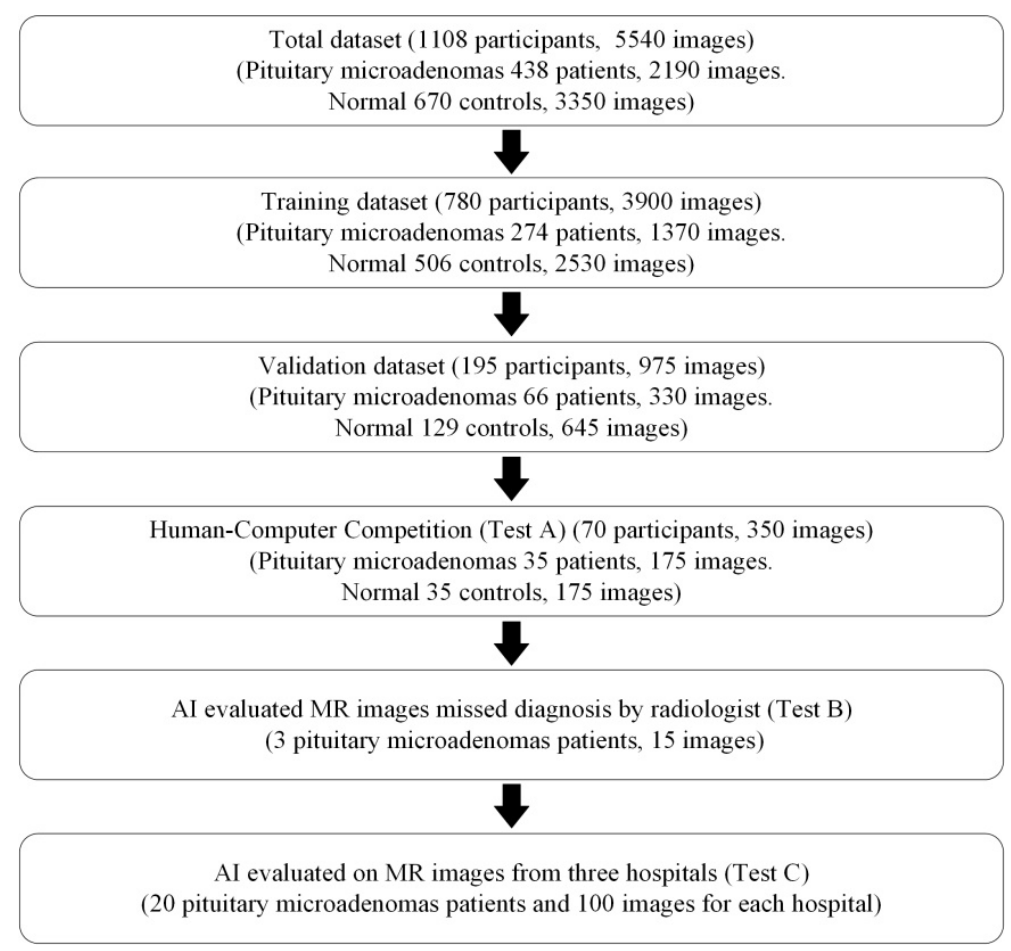


Fig 3

a Human Selection

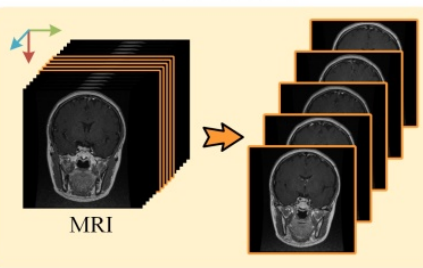

$\begin{array}{ll}\longrightarrow & \text { Axial } \\ \longrightarrow & \text { Sagittal }\end{array}$

$\longrightarrow$ Coronal

7 Convolutional Layers in

Detection Model

7 Convolutional Layers in Diagnosis Model

Computational Operation b Pituitary Detection

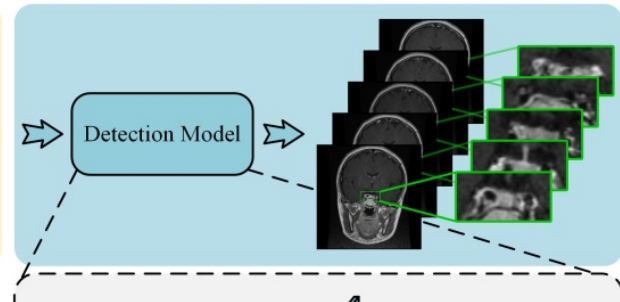

Classification

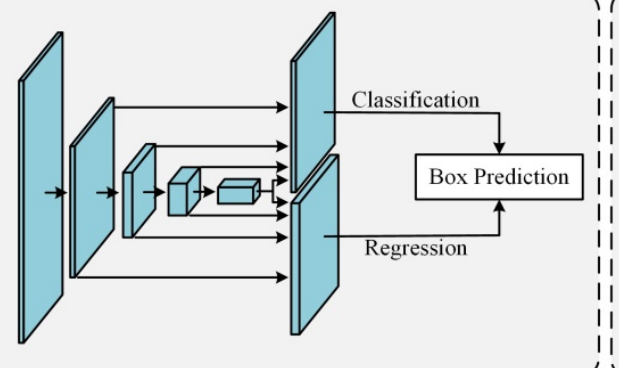

c Microadenoma Diagnosis

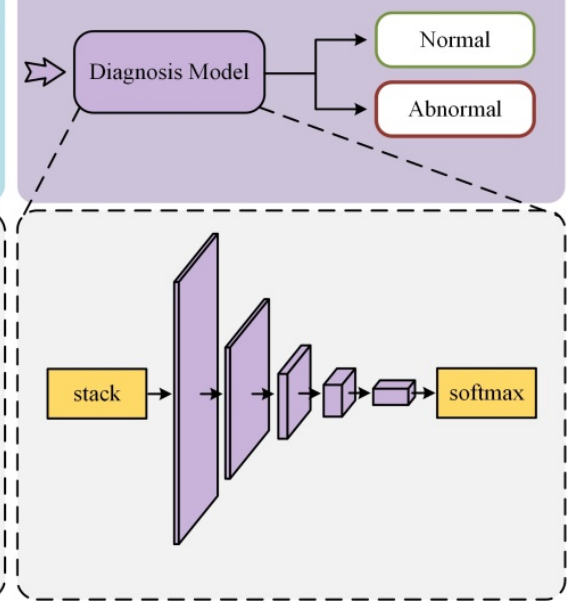


Fig 4

a

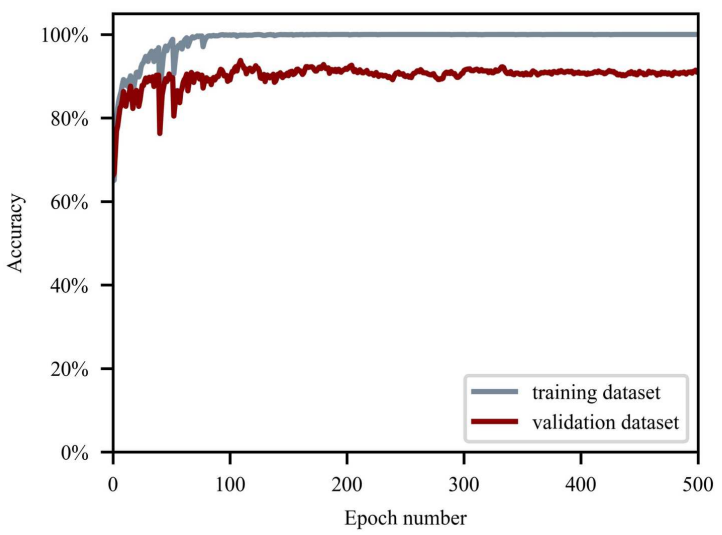

b

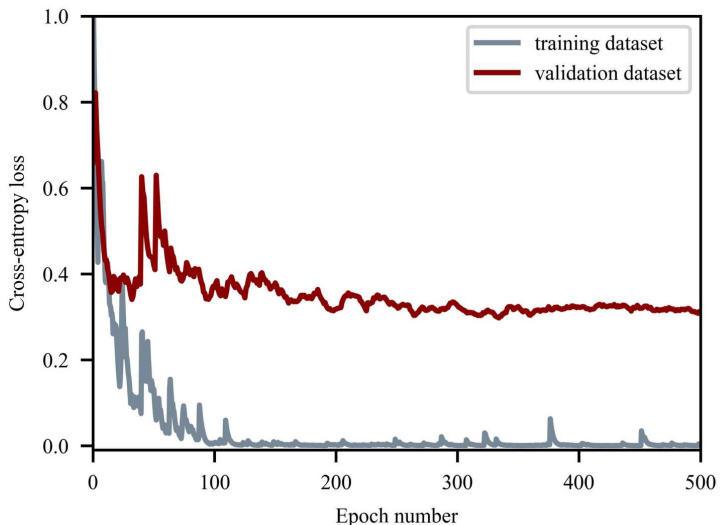


Fig 5

a

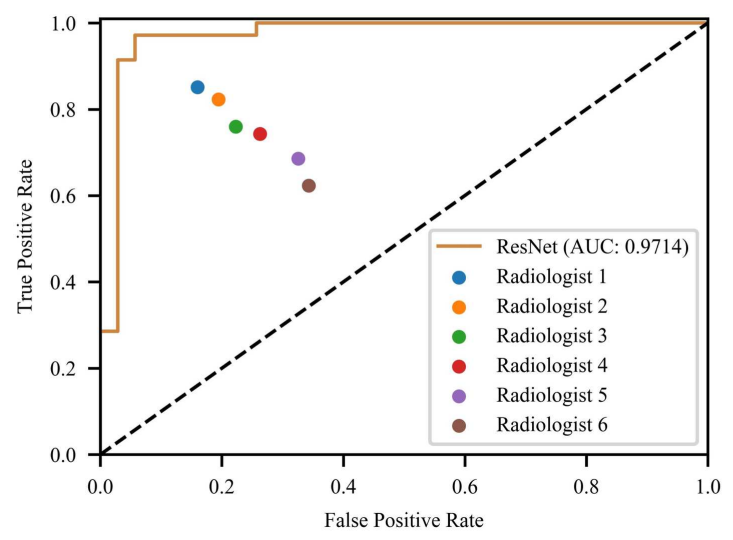

c

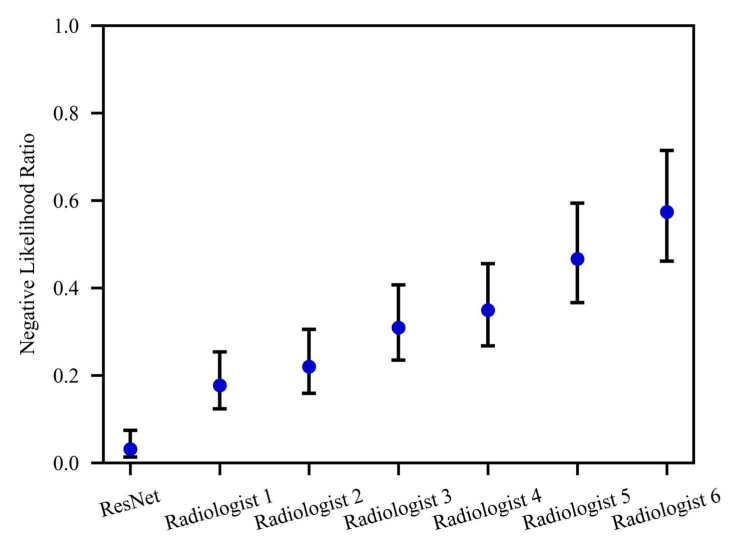

b

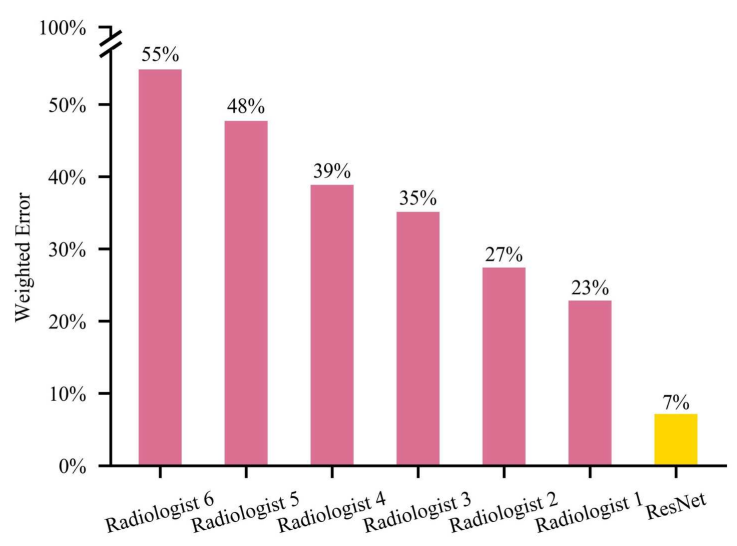

d

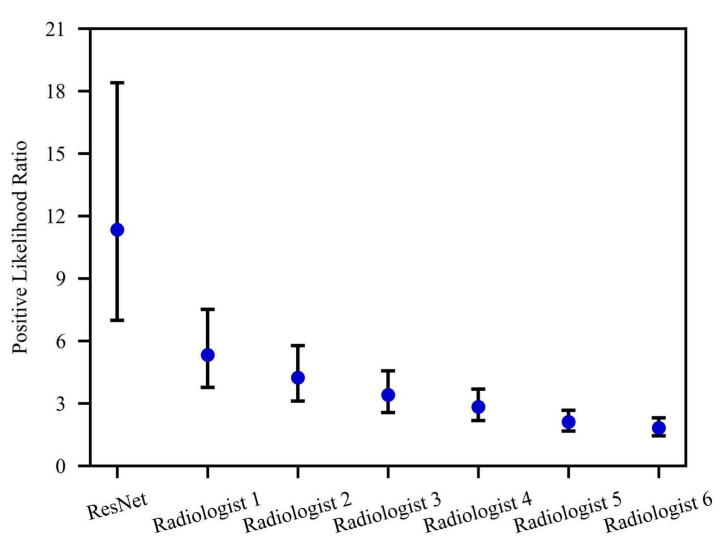


Fig 6

a

Positive cases

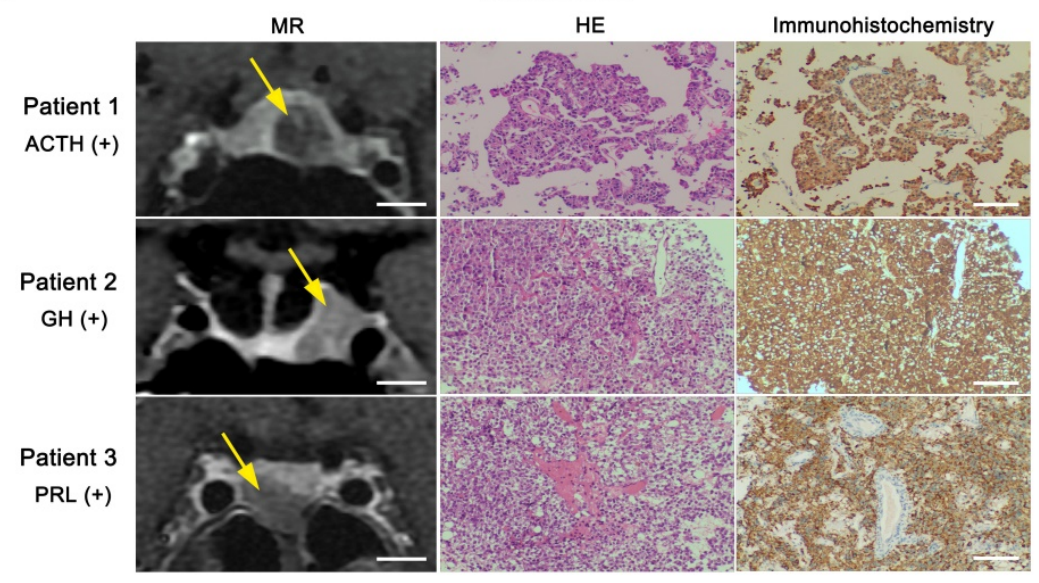

b

False-Negative cases

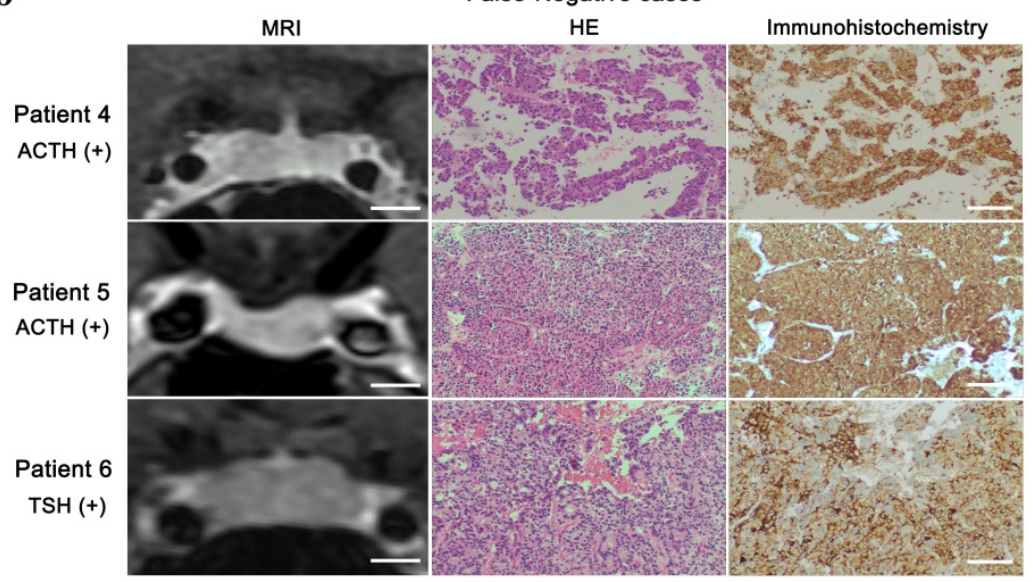


Fig 7

a

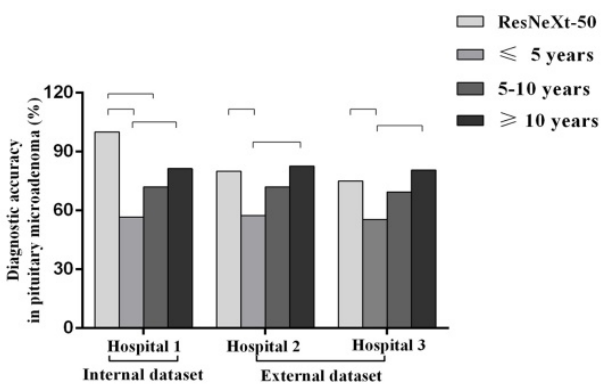

b

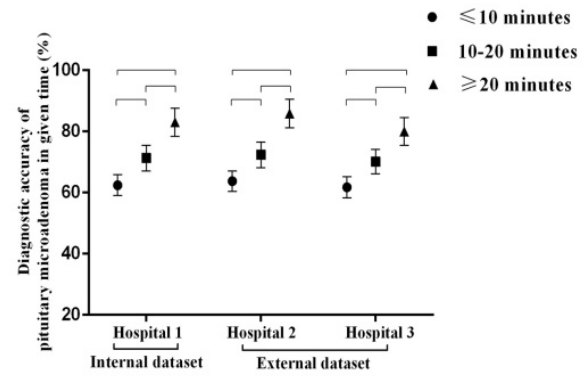




\section{Figures}
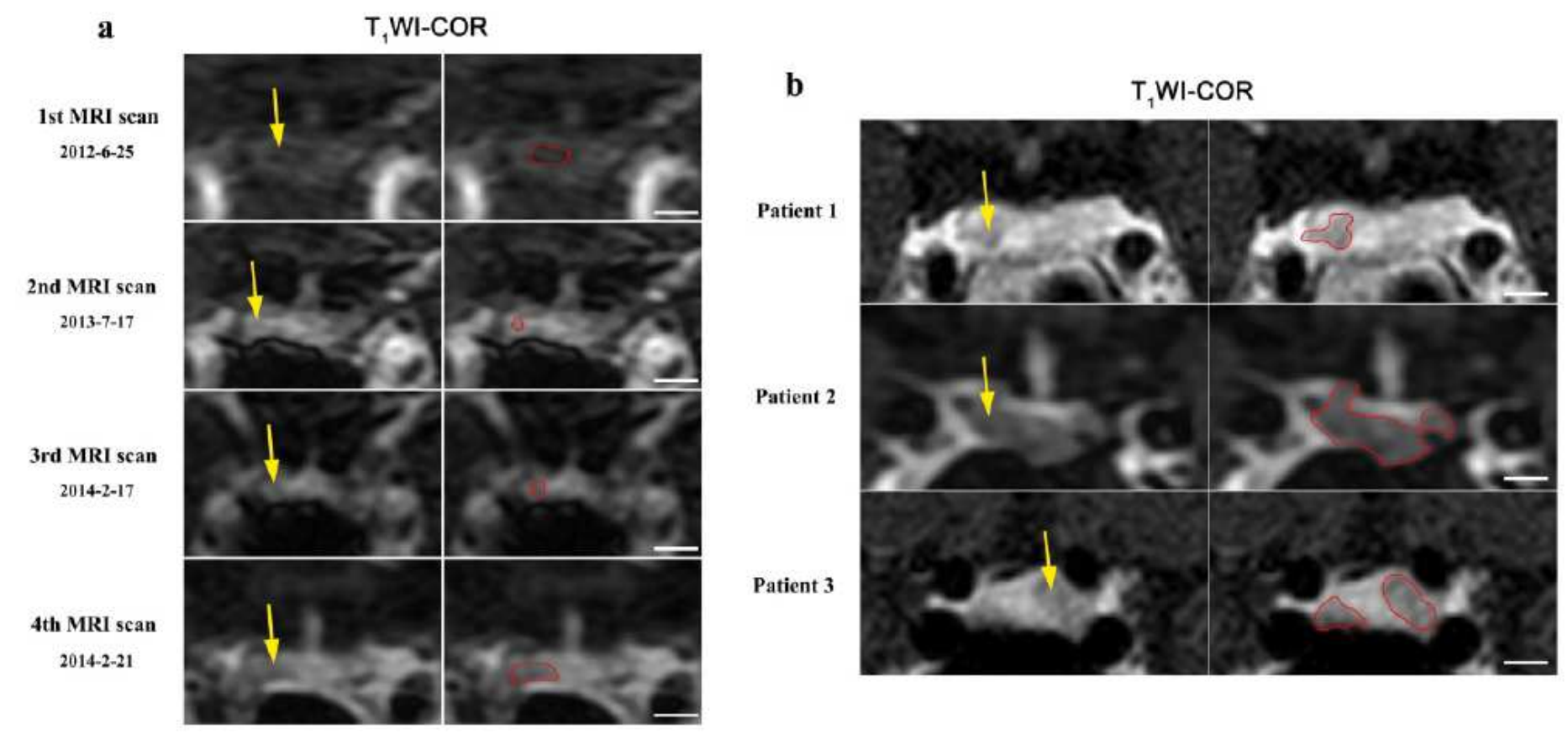

\section{Figure 1}

Representation of misdiagnosed pituitary microadenoma. (a) We showed 4 consecutive pituitary MRI scans over a period of 20 months in a misdiagnosed patient. The radiologist did not pick up the pituitary adenoma during the first 3 times MRI examination. Subsequent ACTH examination of the inferior petrosal sinus localized a functional microadenoma in the region of right pituitary gland. On the 4th MRI scanning, the radiologist detected two pituitary microadenomas. (b) Additional 3 cases of misdiagnosed pituitary microadenoma were shown. Pituitary microadenoma is characterized by variable morphology and prone to misdiagnosis. Patient 1 had a very small pituitary microadenoma with diameter $<3 \mathrm{~mm}$. Patient 2 had an irregularly shaped pituitary microadenoma. Patient 3 had two pituitary microadenomas $(2.8 \mathrm{~mm}$ and $6.1 \mathrm{~mm}$, respectively) and the smaller microadenoma was misdiagnosed. The comprehensive clinical data for patients were listed in supplementary table 1. ACTH: Adrenocorticotropic Hormone. MRI: magnetic resonance imaging. T1WI-COR: T1 weighted imaging-coronal. MRI bar $=5 \mathrm{~mm}$. The yellow arrow and the area inside the red circle are adenomas. 
Total dataset (1108 participants, 5540 images)

(Pituitary microadenomas 438 patients, 2190 images.

Normal 670 controls, 3350 images)

Training dataset ( 780 participants, 3900 images)

(Pituitary microadenomas 274 patients, 1370 images.

Normal 506 controls, 2530 images)

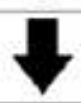

Validation dataset (195 participants, 975 images)

(Pituitary microadenomas 66 patients, 330 images.

Normal 129 controls, 645 images)

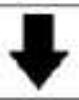

Human-Computer Competition (Test A) ( 70 participants, 350 images)

(Pituitary microadenomas 35 patients, 175 images.

Normal 35 controls, 175 images)

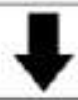

AI evaluated MR images missed diagnosis by radiologist (Test B)

(3 pituitary microadenomas patients, 15 images)

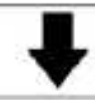

AI evaluated on MR images from three hospitals (Test C)

(20 pituitary microadenomas patients and 100 images for each hospital)

Figure 2

Workflow diagram showing for overall experimental design. All pituitary MRI was performed as part of patients' routine clinical care. There were no exclusion criteria based on age, gender, or race. MRI: magnetic resonance imaging. 

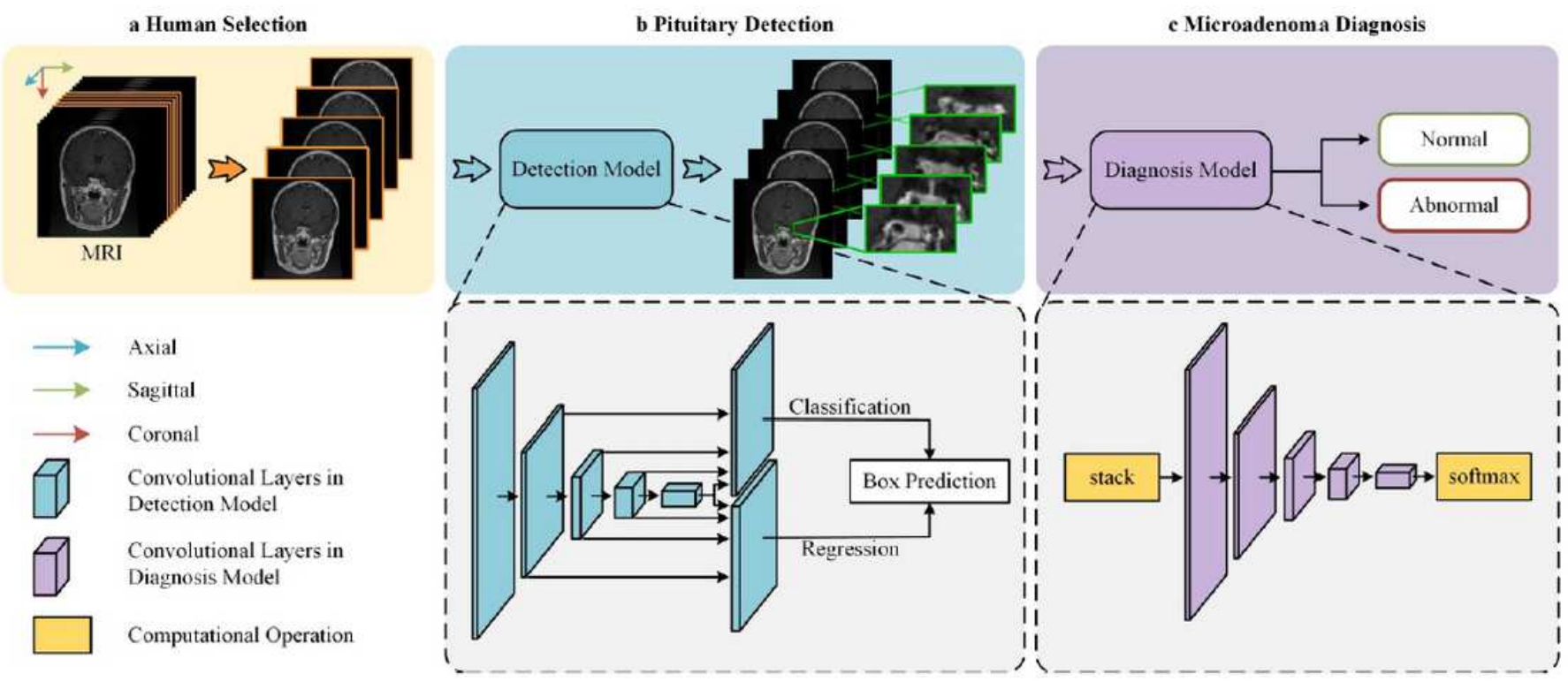

\section{Figure 3}

Overview of proposed CAD system. (a) Five coronal images of the pituitary gland were firstly selected from the original MRI scan by radiologists. Then, the selected MR images were fed into our CAD system for automatic diagnosis. The proposed CAD system consisted two modules: (b) the pituitary detection module employed Faster-RCNN to localize the pituitary region in cerebral MRI. It processed the MRI image using multiple convolutional layers and produced two maps (where the classification map was used to predict the center and the regression map was used to refine the height and width of the rectangle box) for predicting the rectangle box which represented the pituitary region. The pituitary rectangle region was cropped and stacked before feeding into (c) which employed one of CNNs comprising multiple convolutional layers (i.e. VGG, GoogLeNet, ResNeXt, DenseNet, ResNet) to extract the feature. A softmax layer was employed to transform the feature into the presence probability of microadenoma. The images were diagnosed as microadenoma if the $p$ value $>$ or $=0.5$. CAD: computer-aided diagnosis. MRI: magnetic resonance imaging. MR: magnetic resonance. CNN: convolutional neural network. 
a

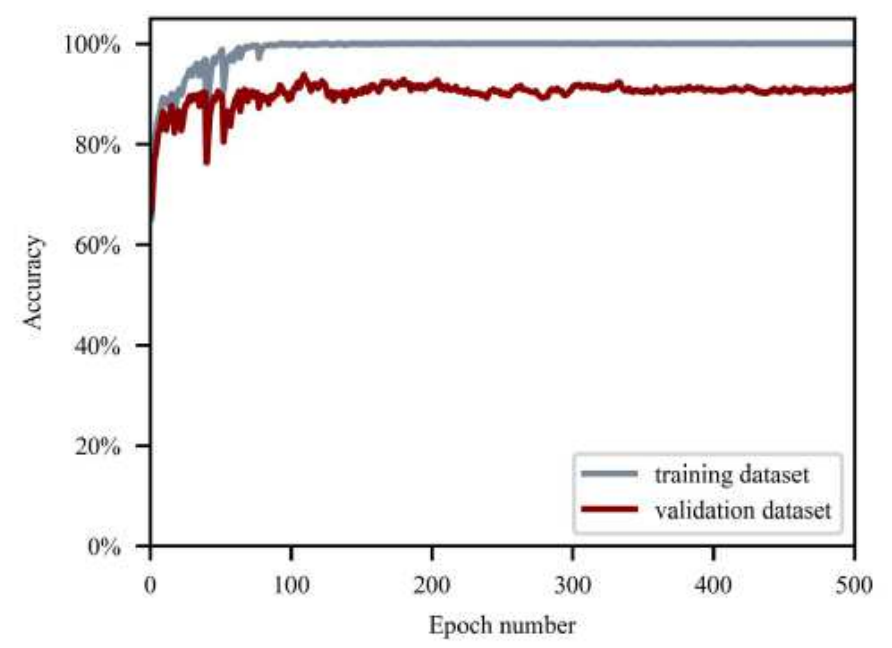

b

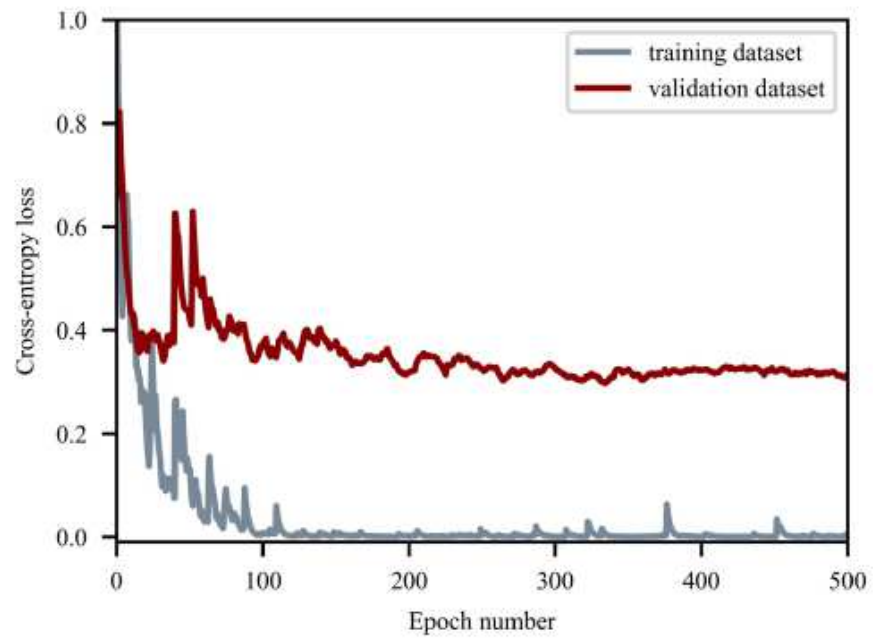

Figure 4

Performance of ResNet model for training and validation datasets. (a). Accuracy curves of ResNet model on training and validation datasets. (b). Cross entropy loss curves of ResNet model on training and validation datasets. After 500 epochs (iterations through the entire dataset), the training and validation datasets were stopped due to the absence of further improvement in both accuracy and cross-entropy. 

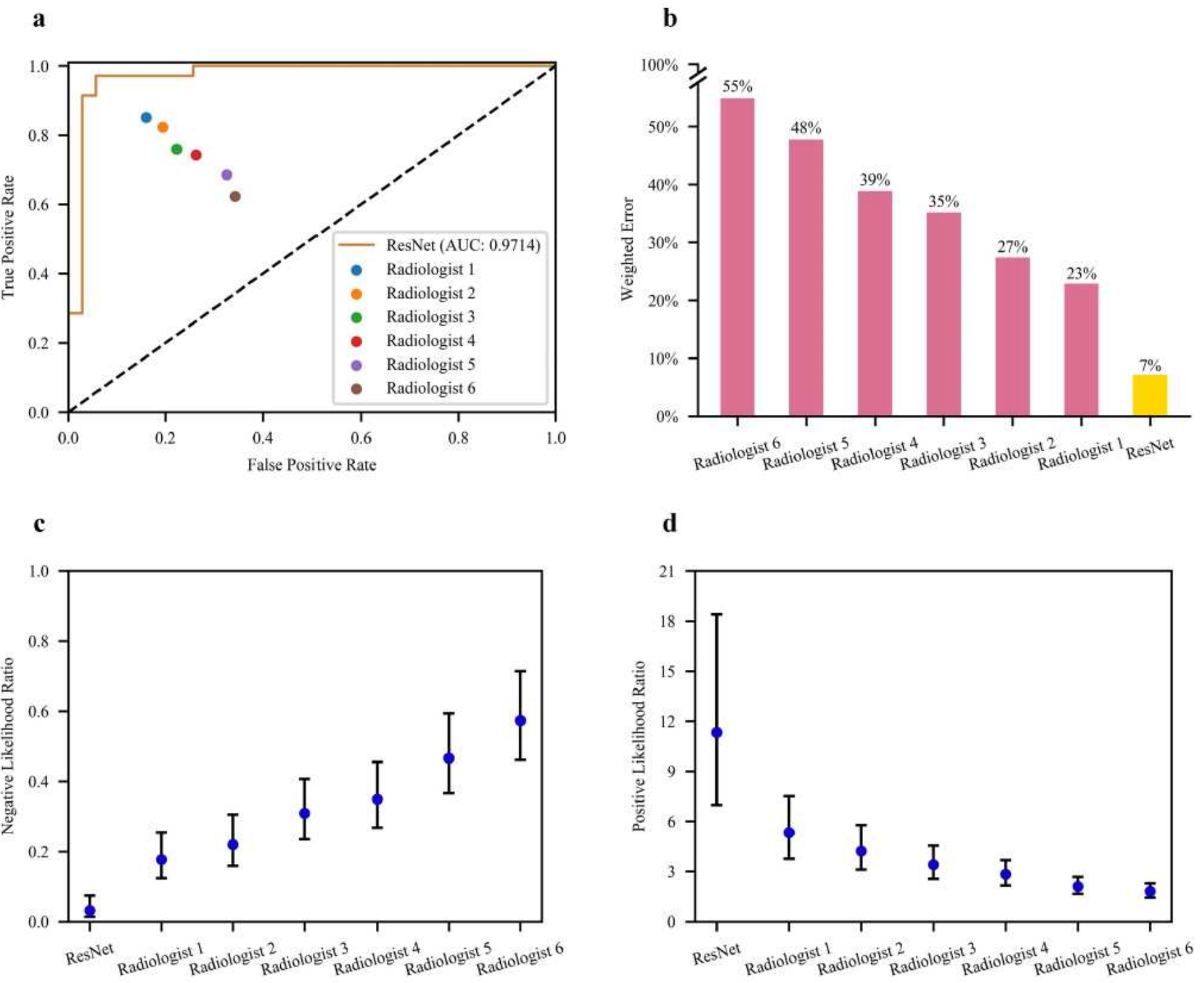

\section{Figure 5}

ResNet model outperformed human radiologists in diagnosing pituitary microadenoma. (a) ROC and AUC: Receiver operating characteristics (ROC) curves plotted the true positive rate (sensitivity) versus the false positive rate (1-specificity). The ROC showed that the ResNet model outperformed 6 radiologists. The AUC (area under ROC curve) of ResNet was $97.14 \%$. (b) Weighted error results based on penalties, the penalties included an error scored of 2 for false negative scored cases and an error scored of 1 for false positive scored cases, the ResNet model yielded a score of $7 \%$ versus the radiologists was $37.3 \%$. (c \& d) The negative likelihood ratio and the positive likelihood ratio: The negative likelihood ratio was defined as the false negative rate over the true negative rate, so that a decreasing likelihood ratio $<1$ indicated increasing probability the absence of microadenoma. The positive likelihood ratio was defined as the true positive rate over the false positive rate, so that an increasing likelihood ratio $>1$ indicated increasing probability the diagnosis of microadenoma. The confidence intervals showed that the ResNet model 
demonstrated statistically better screening performance in both negative likelihood ratio and positive likelihood ratio relative to human radiologists. Radiologist $1 \& 2$ : with $>10$ years professional experience, radiologist 3 \& 4: with 5 - 10 years professional experience, radiologist 5 \& 6: with $<5$ years.

a

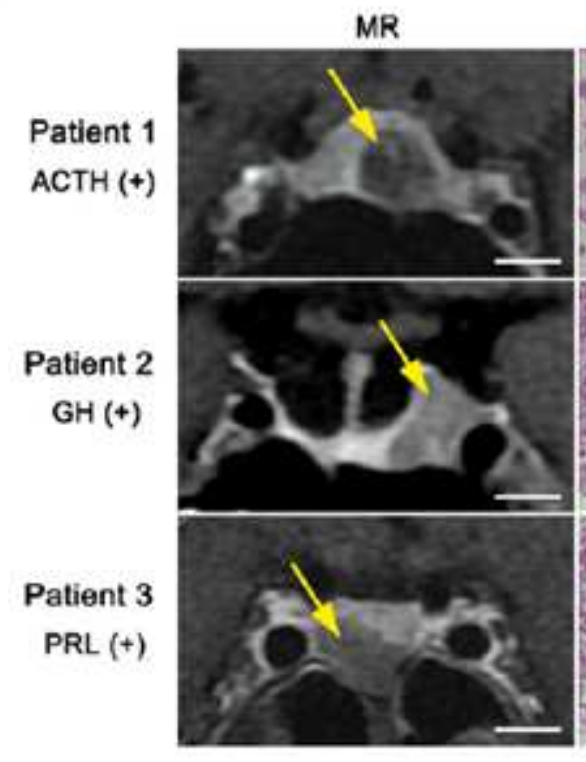

b

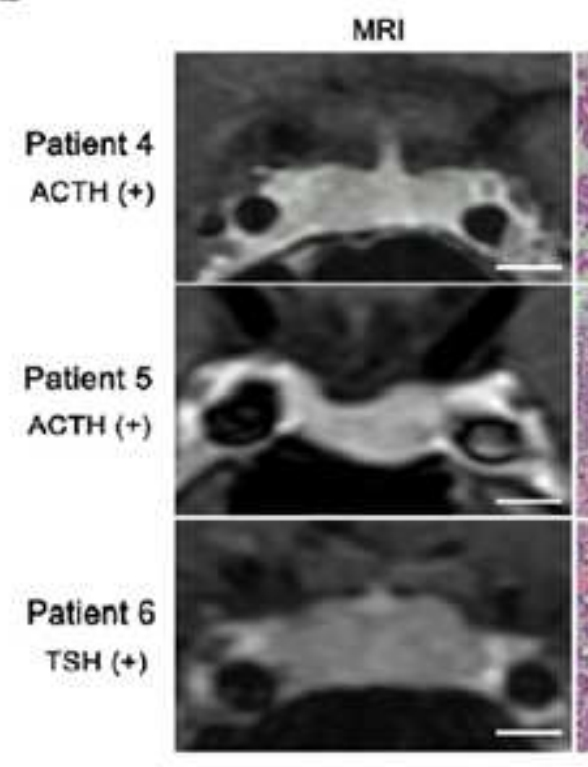

Positive cases

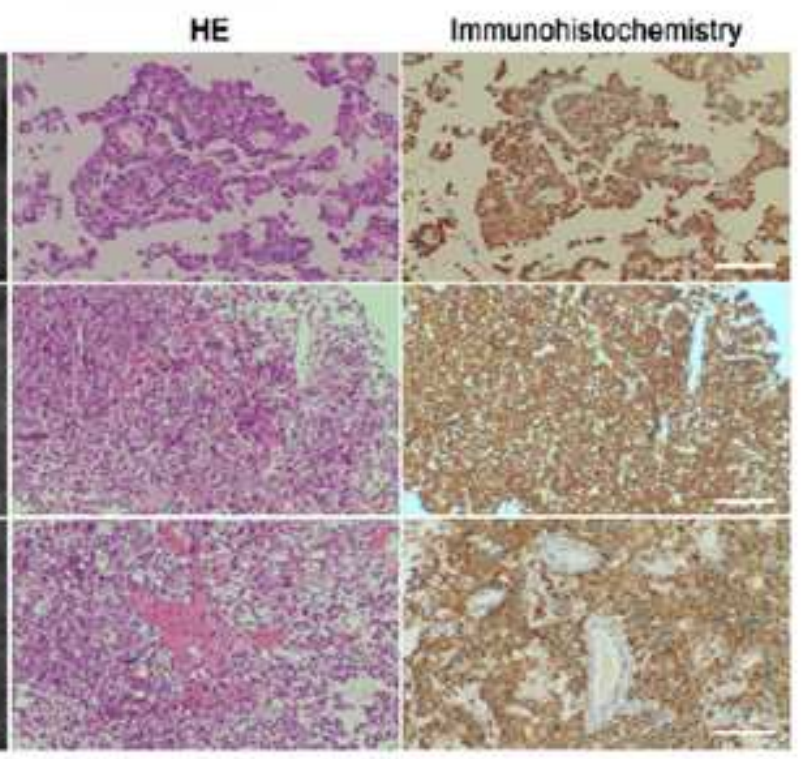

False-Negative cases

HE

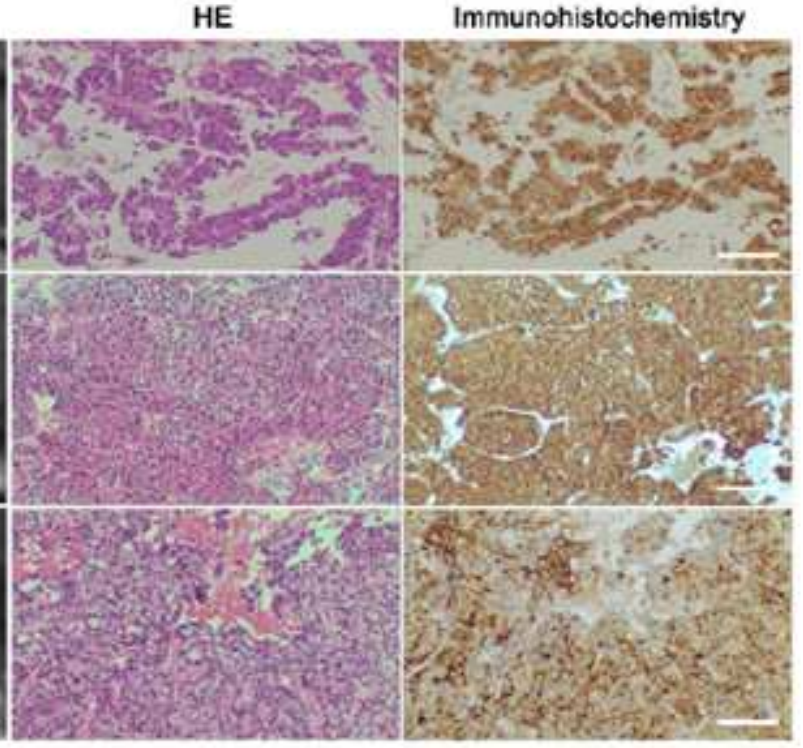

\section{Figure 6}

The MRI and pathologic images of double positive and false negative cases. ( $a, b) 3$ double positive and 3 false negative cases, which were functional pituitary microadenoma, as confirmed by subsequent pathological examination. (b) ResNet model learning 3 false negative cases from radiologists: showing the model diagnosis performance was $100 \%$ accuracy. The comprehensive clinical data for patients were listed in Supplementary table 1. MRI: magnetic resonance imaging. Al: Artificial intelligence. HE: hematoxylin and eosin. ACTH: adrenocorticotropic hormone. GH: growth hormone. TSH: thyroid stimulating hormone. PRL: prolactin. MR bar $=5 \mathrm{~mm}$. Pathology bar $=100 \mu \mathrm{m}$. The yellow arrow indicates a pituitary micro-adenoma. 
$\mathbf{a}$

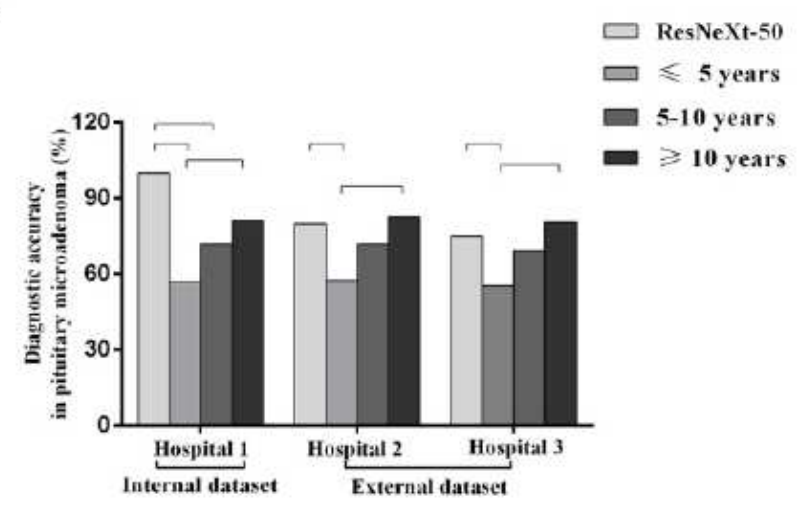

b

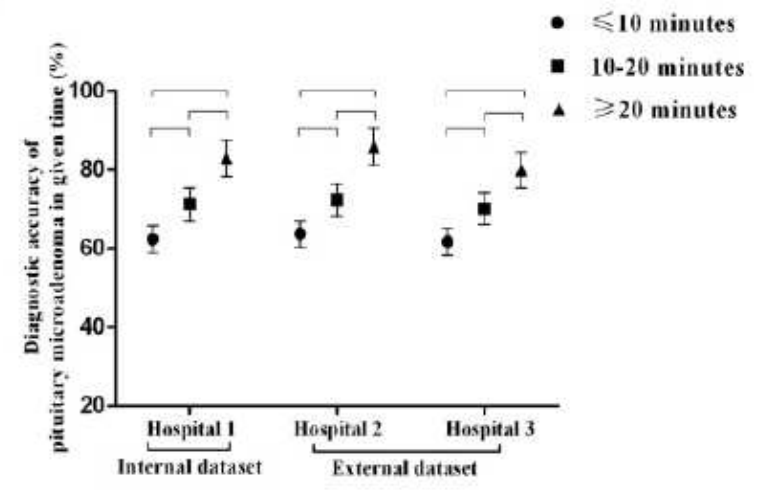

Figure 7

Application of ResNet model in diagnosing pituitary microadenoma. MR images were obtained from 3 hospitals. (a) The diagnosis accuracy rate was significantly increased in senior radiologist and the ResNet model outperformed the radiologists with 5-10 years professional experience in 3 hospitals. (b) The accuracy for diagnosis rate was significantly increased by radiologist with longer reading times. Bar means $p$ value $\mathbb{\text { }}$ or $=0.05$ for comparison of two groups. Al: Artificial intelligence. MR: magnetic resonance.

\section{Supplementary Files}

This is a list of supplementary files associated with this preprint. Click to download.

- microadenomasupplementalinformation.pdf 